\title{
REMOTE ACCESS TO IMAGES AND CONTROL INFORMATION OF A SUPERVISION SYSTEM THROUGH GPRS
}

\author{
Sempere, V. * Silvestre, J. ** Albero, T. * \\ * Dpto. Communications. UPV \\ ** Dpto Computer Engineering. UPV
}

\begin{abstract}
Real time access to control information of supervision systems in large installations is extremely important in order to be able to act in time to critical situations. In this paper the viability of remote access to this information via GPRS is evaluated. Also evaluated are the request protocol image reception and information about the state of the equipment in the system, as well as the average transfer rate in order to establish the update of the information in real time, obtaining update times, for image and/or control data, which are acceptable for a supervision and control system. Copyright (C) 2004 IFAC
\end{abstract}

Keywords: Communication networks, Communication control applications, Control system, Images compression, Supervision.

\section{INTRODUCTION}

In this paper a study is presented on access to control and supervision system information of the purification network of a large city using a PDA through GPRS. The general architecture of the system was presented in a previous article (Sempere, et al., 2003), where the improvements achieved were described, since initially "polling" (Ibe and Trivedi, 1990; Haverkort, 1990) through radio frequency was used for communication between the central and remote stations, presenting poor reliability and low speeds. Currently, through ISDN, the system can exchange control information concurrently, from multiple stations, directly from the sensors and from the installation, or point to point from whatever control equipment (PLC, terminal, etc). It can also capture images of critical zones within the installation and transmit them to the central station where they are processed.

The paper is structured in the following way: In section 2 can be found a general description of GPRS technology. In section 3 the simulation environment and the protocol for image request from the PDA is described briefly. Some measurements are shown in section 4 , and in section 5 the conclusions obtained are presented.

\section{GENERAL PACKET RADIO SERVICE} (GPRS)

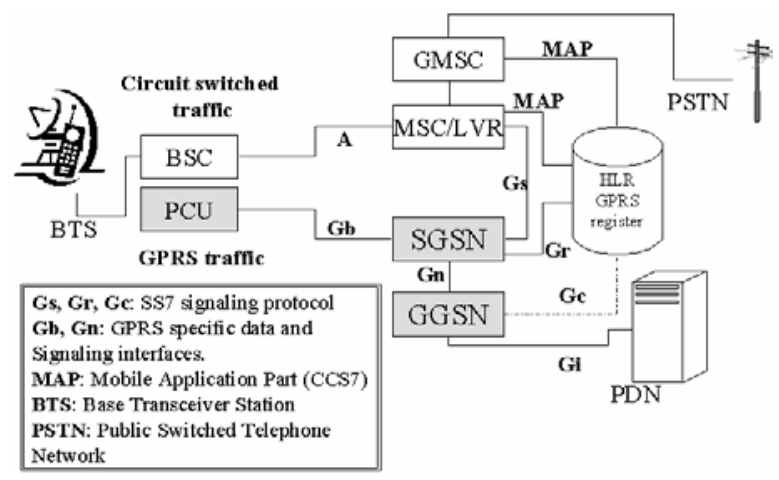

Figure 1 GPRS network architecture

GPRS is an extension of GSM mobile communication technology. GPRS has been designed for applications that go further than just voice, who known as Advanced Services in Information Mobility. GPRS uses the existing GSM network and adds two new packet-switching network equipment: GGSN (Gateway GPRS Support Node) and SGSN (Serving GPRS Support Node). In figure 1, it can be seen that GPRS introduces at the BSC level (Base Station Controller) the unit known as PCU (Packet Control Unit).

GGSN is similar to the GMSC (Gateway Mobile Services Switching Center), it acts as a logical interface toward the external PDN (Public Packet Data Network) or other GPRS networks. The GGSN connects with the HLR (Home Location Register) by means of the Gc interface. The SGSN controls the connection between the network and the mobile 
station (MS), and is in charge of the delivery of packages to the mobile terminal in a way similar to that of the MSC (Mobile Switching Center) and the VLR (Visitor Location Register) made in the GSM. The following are characteristics of the GPRS:

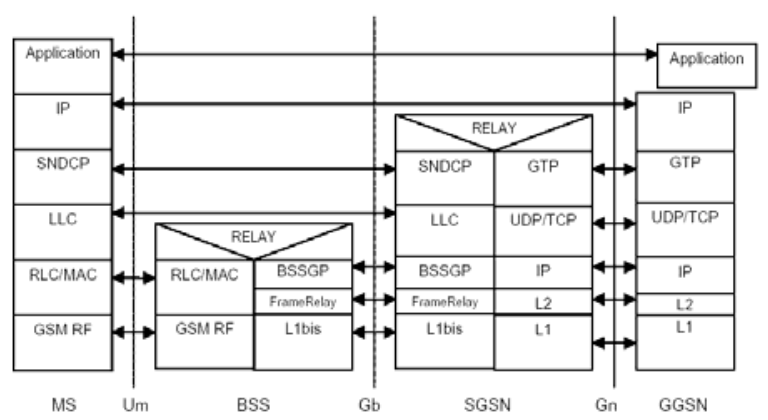

Figure 2 GPRS protocols

- It is an always-on service. Users only pay for the amount of data they transfer rather than for the length of time they are connected to the network.

- It meets the Internet communications protocol (IP).

- It uses advanced codification with different classes and greater speed than GSM.

- It allows linking of time intervals to increase capacity.

- The GPRS system is completely compatible with voice transmission via GSM.

\subsection{The GPRS Protocols Layers}

Figure 2 shows each one of the protocols that intervene in the transmission of data and signalling information in GPRS. Following this, there is a brief description of each protocol. [Agilent Technologies, 2001; Ericsson, 2002, Ghribi and Logrippo, 2000; Granbohm and Wiklund, 1999]:

- GTP (GPRS Tunneling Protocol): Receives IP datagrams and sends them to the GPRS support nodes.

- TCP/UDP (Transmission Control Protocol and User Datagram Protocol): TCP is used to transfer Protocol Data Units (PDUs) with reliability and UDP is used to carry the information that do not require reliability.

- IP (Internet Protocol): This is in charge of routing user data and signalling information across the Gn interface.

- SNDCP (SubNetwork Dependent Convergence Protocol): This protocol is used to convert the network layer PDUs $(\mathrm{N}$ PDUs) into an appropriate format for the GPRS architecture.

- $\quad$ LLC (Logical Link Control): This protocol obtains a reliable logical link between the SGSN and the mobile phone.
- BSSGP (Base Station System GPRS Protocol): Sends information between the SGSN and the BSS.

- NS (Network Service): Uses frame relay across the $\mathrm{Gb}$ interface. It could be used like a point to point connection between the SGSN and the BSS or as a frame relay network.

- RLC (Radio Link Control): Transfers LLCPDUs between the layer an the MAC function, segments the LLC-PDUs into RLC data blocks, segments and regroups the $\mathrm{RLC} / \mathrm{MAC}$ messages into RLC/MAC control blocks.

- MAC (Medium Access Control): This protocol controls the access signalling across the air interface.

\subsection{Data transfer}

Having defined the different GPRS protocol layers, how packet data units (PDUs) are transmitted (Fabri, et al., 2000; 3GPP, 2002; Inacon, 2000) is going to be described. The data has to travel from the Internet to the GGSN, then to the SGSN, to the BSS, and finally to the MS (in this paper the PDA). Along the way the data suffers a series of changes, ie, headers are added in each of the layers, also occurring are segmentation and even compresion if necessary.

In figure 3 data flow is shown and it is possible to appreciate that is rather header-heavy, therefore the available physical link layer throughput is occupied by header bits, rather than information data.

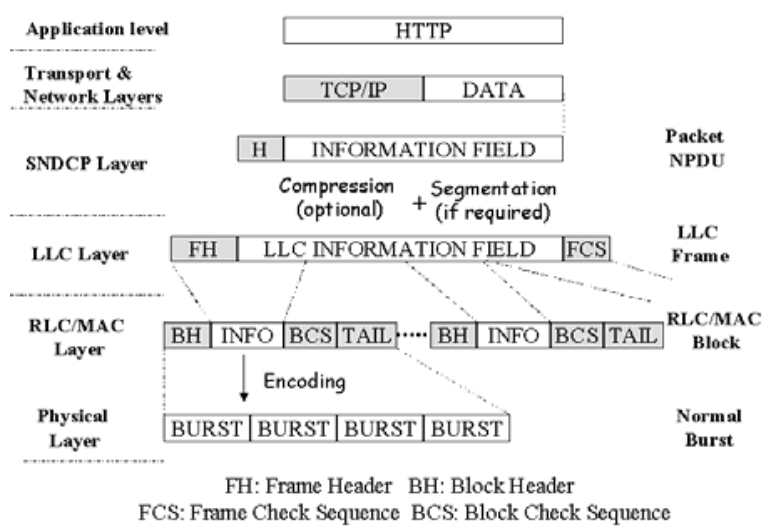

Figure 3 GPRS Data Flow

The SNDCP is responsible for a number of functions: compression and decompression of user data, compression and decompression of packet headers (this function is only applicable in case of TCP/IP), segmentation and desegmentation of N-PDUs, (Inacon, 2000).

The LLC layer concatenates the radio blocks to/from the RLC/MAC layer into larger segments (LLC frames). A LLC frame has a variable length and consists of a header (address (1 octet) + control field (1-36 octets)), an information field with the user data 
to/from the upper layers (140-1520 octets), and a Frame Check Sequence field (FCS, 3 octets), (3GPP, 2002).

The RLC/MAC data is transmitted in radio blocks that use a structure with a MAC header. There are two types of blocks: the control blocks, with signalling information and the data blocks, with user data. The size of the radio blocks depends on the Coding Scheme (CS) used in the transmission, a control block is always coded with CS-1 while data can be coded using any of the four coding schemes, (Ericsson, 2002). In this paper the CS-2 which uses the PCMCIA GSM/GPRS card installed in the PDA is going to be considered. The RLC/MAC block is composed by the header block (MAC header ${ }^{1} 1$ octet, RLC header 2 octets), some spare bits added (7 bits) it is a padding needed to adapt the radio block size to the channel coder, the RLC payload data block (30 octets), the Block Check Sequence (BCS, 16 bits) and after all a tail bits (4 bits). Each radio block is assembled differently depending on the coding scheme used, however, regardless of coding scheme, each radio block must be made 456 bits long in order to fit it into the GSM bursts.

\section{EXPERIMENTATION ENVIRONMENT.}

The system in which the tests have been carried out is made up of 3 fundamental parts, see figure 4 . For a detailed description of the complete system see Sempere, et al., 2003:

\section{- Central station. \\ - Remote stations. \\ - Communication network.}

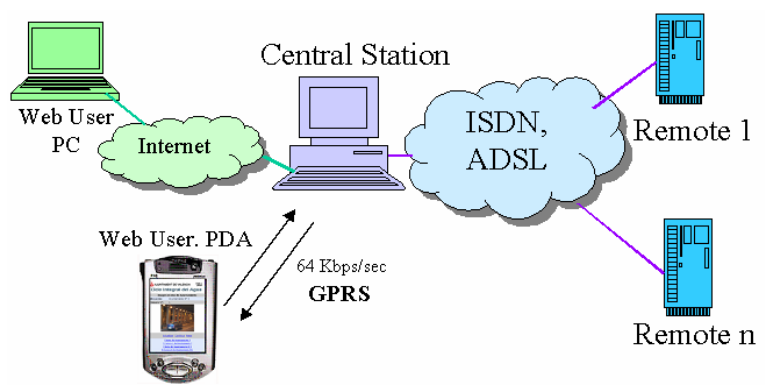

Figure 4 Scheme of the simulation environment

\subsection{Central station}

This is the part charged with managing the information coming from all the installation and controlling the remote communication between the central station and the remotes. It communicates simultaneously (using several connections TCP/IP) with all the remote stations.

\footnotetext{
${ }^{1}$ In the byte which forms the MAC header 3 bits correspond to the USF (Uplink State Flag), this is precoded with a block code in the CS-2, CS-3 and CS-4 in order to detect any errors in it. Therefore the precoded USF is 6 bits and the MAC header for the CS-2 is (8-3)+6=11
}

The central station uses equipment which does the SCADA (Luque, et al., 1996) in the new network and houses the state and image data base. A background process is charged with preseving coherence between the old data base (updated by polling) and the new one.

Local application of the central station. In the application of the central station is found a map of the city where the different stations are located, when being placed on a station a pull-down menu appears with the options to execute.

The parameterization of the variables to read (states) and to write (orders) from the PLC is made in the central station. In this configuration the position in the zone of memory or marks that will contain the order/state information is indicated. The initial values of the states zone are stored in the data base when finishing the parameterization and later, each change that is detected is stored. Another option is to visualize the states of the equipment of a remote station and can give orders from the central station.

The images are visualized in the central station in real time connecting itself to a remote station, if the operator considers it necessary he can store them. Another type of storage is that made in background. The parameterization of the images is made and the recording is made with a constant regularity throughout the day. Later the stored images can be recovered (background or manual).

Web application of the central station The central station also houses a Web server which permits the data base which registers information from each remote to be consulted by Internet.

Access to the information can be made from fixed or mobile nodes using GPRS. For this a Web page has been designed which in the moment of connection detects and adapts to the type of user whether it is fixed (PC) or mobile (PDA). The application has been developed with JavaScript, VBScript, ASP and HTML. For PDA JavaScript 1.0 is used due to superior versions not being accepted.

The application Web security is one of the important aspects. For this there is a system of authorized users which restricts open access. Moreover a Web user can only consult information which has no control over the system.

If the client connects from PC, he is permitted to see control data and images in real time, and stored images. The client can gain access to this information in two ways, either by way of a tree-control, or through the interactive map that calls up a menu. The real time images are updated in a fixed time and it is possible for the client to capture an image that interests him and store it. The control data option has the task of showing the state of the equipment that makes up each station. 
If accessed through a PDA client, the images can be seen live, as well as the information on conditions in real time, Fig. 5. PDA, Compaq iPAQ 3970 has been used.

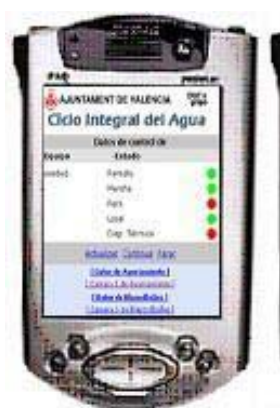

a)

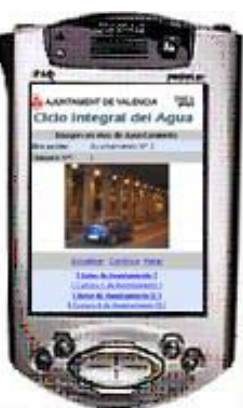

b)
Figure 5 Web Application for PDA client. a) visualization of control data, b) visualization of images in real time.

The decision to create a Web application is determined by the elevated price of a point to point GPRS connection with the central station or with the remotes through which the mobile users access information. For this reason, it has been decided to use the Internet as a transport network and GPRS as access technology.

\subsection{Remote station}

It can capture information proceeding from different cameras situated in the installation, then process and transmit it together to the central station by means of the communication available in each case. It is capable of communicating bi-directionally, with any PLC on the market (the Simatic S5/S7 protocol has been used in the prototype) and mapping the variable principles of the process in a simple way. The process of the remote station determines when variations have been produced that are susceptible to being sent to the central, carrying out an automatic update.

Application of the remote station There are three process: "CapturaImag", "ClienteImag" and "ComprImag". The first is the task of capturing the images from the camera and sending them to "ComprImag", which compresses them. "ClienteImag" transmits them to the central and also receives the parameterisation information. The three processes share a common memory zone that allows them to communicate in a synchronised manner. The central carries out the parameterisation and indicates when the transmission/reception can be initiated or delayed, using messages to the "ClienteImag" process.

The communication of the remote with the PLC is carried out using the PlcS5 ActiveX Control, which uses the Siemens Simatic S5 AS511 protocol over a series link. In the case of a change taking place in the
PLC, an alarm signal is sent from the remote station to the central station.

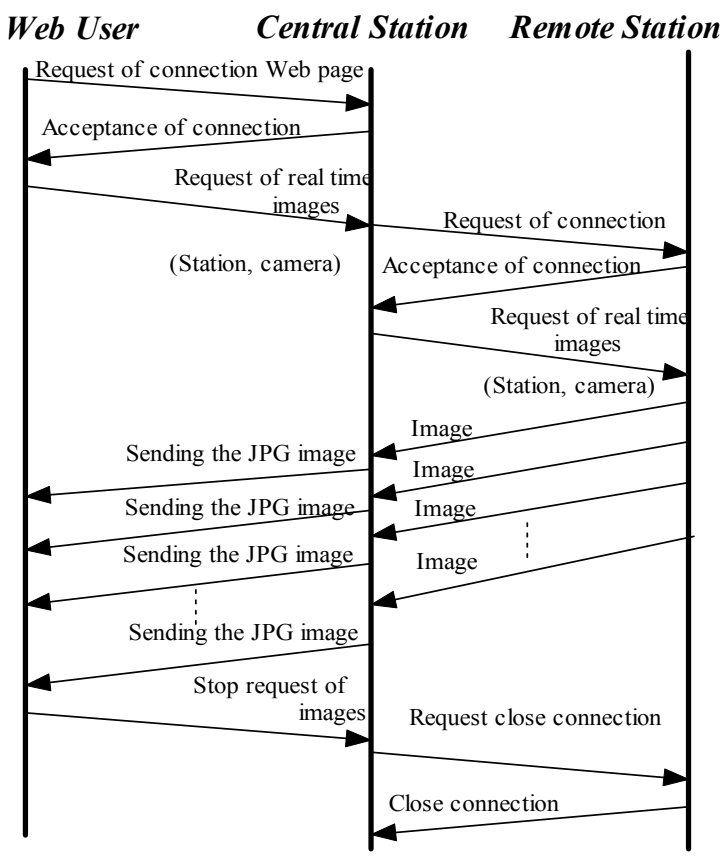

Figure 6 Protocol for the request and reception for a Web user.

\subsection{Communications network}

This gives communication support to all the remote stations, permitting the incorporation of services in real time such as the transport of images, states and orders. The previous system of polling using radio frequency has become a redundant back up system, which operates in the case of failure with minimum service.

Three alternatives have been studied for the interconnection of the central station with the remotes: a) Use of point to point connections through the RDSI (Intergrated Services Digital Network), b) VPN (Virtual Private Network) (Kosiur, 1998) through the Internet with RDSI access and c) VPN through the Internet with ADSL access. GPRS has been used for the interconnection between the central station and a mobile Web user (Hoymann and Stuckmann, 2002).

\subsection{Protocol for the request and reception of images via GPRS}

Once the environment in which the tests have been carried out have been generally described, the protocol for the request and reception of images is described, figure 6 , where the finished study is centred. This same protocol is similar to the one carried out in the request of a Web user to visualise the PLC states.

When a Web user wants to seek information on a remote, whether on PLC states or images, he must be connected to the Web page and be identified. Once 
the access to the Web page has been permitted, the request can be made. This request is picked up by the local program of the central station through a message sent by the Web application.

The central station then requests connection to the remote station so as to offer the information in real time to the Web user. When the connection is established, the remote station sends the requested information to the local application of the central. In the case of states, if there are changes, they are stored in the database, and in the case of images, these are compressed creating JPG files, which use the PDA to visualise the requested images.

\section{EXPERIMENTS AND RESULTS}

The characteristics of the PCMCIA GSM/GPRS Xacom card used are the following:

- Class $12 \mathrm{SW}$ (4+4, 5 simultaneous).

- Transference velocity $67.0 \mathrm{Kpbs}$ in Coding Scheme 2.

The measurements of the transference rate were made using two different methods, obtaining very similar results in both. The first method used was the connection using GPRS to a Web page that measures the transference speed (Kbps). The steps to carry out the measurements are the following: A primary estimation of the speed is made sending a page of a determined size. Measuring the time taken to download the page, a primary result of the speed of download is obtained. Secondly, the real measurement is carried out by sending a page of an appropriate size at the speed calculated in the previous stage. In the second method an FTP server is used. A file of fixed size is downloaded from the ftp client of the PDA, and the time and size of the download is obtained, calculating the rate in Kbps.

Measurements were also taken of the transference rate on different days. In figure 7 the variability of the transference rate $(\mathrm{Kbps})$ over a period of 5 days is shown, where the measurements have been taken between 9:00 hrs and 18:00 hrs with intervals of 15 minutes in the city of Alcoy. Also, the deviation from the average with respect to the maximum and the minimum of each instant of measurement is rather high. Measurements were also taken to obtain the transference rate in three other cities and in different time zones $(13: 00-20: 00,20: 40-23: 45,7: 30-$ 9:00), figure 8 .

According to figures 7 and 8 , and table 1 where the average speed obtained from the different measurements is shown in the area where the tests have been carried out, the average speed which is indicated in the characteristics of the card is not reached. As is known, the transference rate as well as the characteristics of the card depend on the following: GSM users who are using voice, the GPRS users who are connected at that moment, the geographical area, and last but not least, the operator who offers the service, in the case of the tests, Telefónica Movistar.

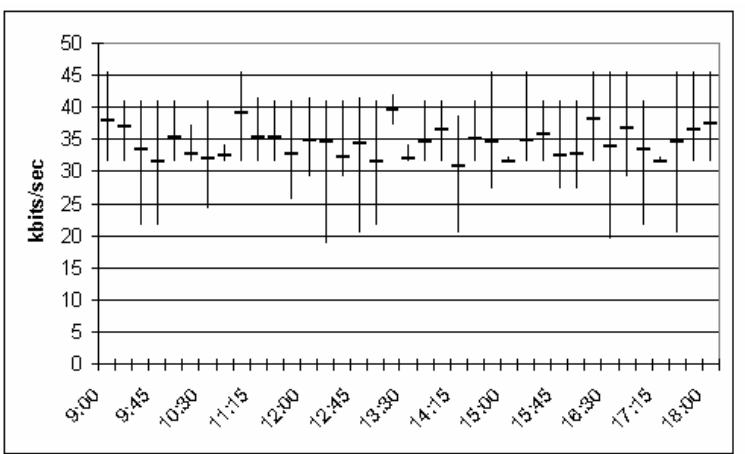

Figure 7 Graph which shows the average, maximum and minimum value, over a period of five days for each instant of measurement, between 9:00 and 18:00.

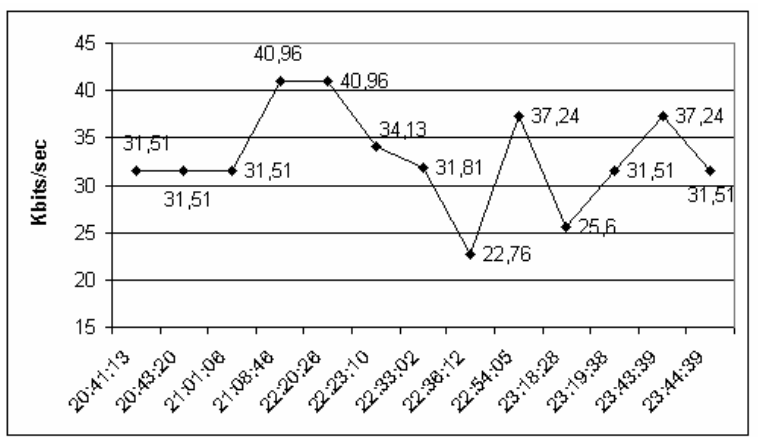

Figure 8 Transference rate obtained with measurements taken between 20:40 and 23:45 for a single day.

Tabla 1 Average value of the transference rate $\underline{(\mathrm{Kbit} / \mathrm{s})}$

\begin{tabular}{c|ccccc}
\hline City & Day1 & Day2 & Day3 & Day4 & Day5 \\
\hline Alcoy & 33.5 & 30.67 & 36.07 & 35.73 & 36.19 \\
Valencia & 34.69 & 34.30 & 32.41 & 36.06 & 35.35 \\
Banyeres & 35.87 & 32.94 & 38.21 & 36.48 & 35.89 \\
Játiva & 32.62 & 36.79 & 35.90 & 31.72 & 33.52 \\
\hline
\end{tabular}

Bearing in mind that the JPG image that is visualised on the PDA Web page has a size that varies between 4 and 7 Kbytes, the time to download the image, taking an average rate of $34.46 \mathrm{Kbits} / \mathrm{sec}$, varies between $0.91 \mathrm{~s}$ and $1.63 \mathrm{~s}$ (Tu, Unloading time). Also, an Internal Processing Time (TIP) exists, in which the defragmentation and decompression tasks are included for the visualisation of the image on the PDA. This can last between 1 and $1.5 \mathrm{~s}$. Another time to bear in mind is that which the user needs to be able to observe the image (Tv, Visualisation Time); which various observers believe should not be inferior to $3 \mathrm{~s}$.

Another aspect to consider is the following: due to the special characteristics of wireless links when TCP protocol is used in GPRS networks, it may not be able to achieve expect performance (Gurtov, et al., 2002). The wireless links are prone in general to a higher transmission error rate (interference, fading and poor signal quality, packet loss contributed to node 
mobility). TCP incorrectly interprets packet loss or delay as the results of congestion, then TCP proceeds to congestion control and the throughput is affected. For this reason, a propagation delay $(\Delta \mathrm{T})$ is considered between 0.066 and $200 \mathrm{~ms}$ (Zhou, et al., 2002).

Considering the worst case, the time (T) that must be established for the updating of the image on the PDA web page follows the expression below:

$$
\begin{gathered}
\mathrm{T}=\mathrm{T}_{\mathrm{U}}+\mathrm{T}_{\mathrm{IP}}+\mathrm{T}_{\mathrm{V}}+\Delta \mathrm{T} \\
\mathrm{T}=1.63+1.5+3+0.2=6.33 \mathrm{~s}
\end{gathered}
$$

If this same time is calculated for the best case, the result obtained is $4.91 \mathrm{~s}$. However, different tests have been carried out with this time, and in many of them it has not been sufficient for the image to be shown on the screen. With a time of $6 \mathrm{~s}$ the images are visible and the observer can analyse them. Therefore, this updated time has been finally established for the image shown on the PDA.

\section{CONCLUSIONS}

A viable system has been presented to which one can gain access via GPRS to obtain control information (states of equipment and images of the remote stations in real time) at any time of day.

As regards the images in real time, the visualisation time Tv, which the user has available to observe the image, can be inferior or superior to the time considered necessary (3s). This is due to the fact that the variation in other times for various reasons (congestion, transference speed, errors in transmission, etc.) has repercussions on the value of Tv. The user, therefore, sometimes does not have the necessary time available to observe the image on the PDA completely.

The total time necessary for the update of information about states in real time is $4 \mathrm{~s}$, which is inferior to that necessary to see images in real time. This is due to the fact that the information about a change in state contains little data to transmit, so the reception of the said values is faster, the possibility of error is smaller and also the time necessary for observation on screen is shorter.

It has been confirmed that the Telefónica Movistar operator offers an average rate of $34.46 \mathrm{Kbits} / \mathrm{sec}$ and this never goes above $40.96 \mathrm{Kbits} / \mathrm{sec}$.

In later studies, an analysis of the behaviour of the system is intended, using another GPRS operator such as Amena or Vodafone. After analysis, the operator that offers the greatest transference rate will be chosen, to shorten the refreshment time established for both images and states.

In future studies it is also intended to provide greater security for the PDA Web application, so as to have control over the system. That is, to be able to give orders to the remote and to position cameras, and not to only obtain information as happens now.

\section{REFERENCES}

Agilent Technologies (2001). Understanding General Packet Radio Service (GPRS). Application Note 1377. http://agilent.com

Ericsson (2002). GPRS Measurements in TEMS Products. White paper http://www.ericsson.com/services/tems/whitepapers .shtml

Fabri, S.N., S. Worrall, A. Sadka and A. Kondoz. (2000). Real-Time Video Communications over GPRS. 3G Mobile Communication Technologies, Conference Publication No. 471. pp. 426-430.

Ghribi, B. and L. Logrippo (2000). Understanding GPRS: the GSM packet radio service. Computer Networks 34, pp. 763-779.

Granbohm, H., J. Wiklund (1999). GPRS General Packet Radio Service. Ericsson Review. No 2.

Gurtov, A., M. Passoja, O. Aalto and M. Raitola (2002). Multi-layer Protocol Tracing in a GPRS Network. IEEE Fall VTC.

Haverkort B. R. (1999) Performance Evaluation of Polling Based Communication Systems Using SPNs. In: Application of Petri Nets to Communication Networks:Advances in Petri Nets (J. Billington, M. Diaz and G. Rozenberg, Eds) 1rd ed.. pp. 176-209. Springer. Berlin.

Hoymann, C. and Stuckmann, P. (2002) On the Feasibility of Video Streaming Applications over GPRS/EGPRS. IEEE Global Telecommunications Conference.

Ibe, O.C. and K.S. Trivedi (1990). Stochastic Petri Net Models of Polling Systems. IEEE Journal on Selected areas in Communicatios, Vol 8, No 9, pp. 1649-1657.

Inacon. (2000). GPRS From A-Z. http://www.inacon.com

Kosiur, D. (1998) Building and Managing Virtual Private Network. Wiley.

Luque, J., J.I Escudero. and I Gómez. (1996). Determinig the Channel Capacity in SCADA Systems Using Polling Protocols. IEEE Transactions on Power Systems. Vol. 11, No. 2, pp. 917-922.

Sempere, V., T. Albero and J. Silvestre (2003). Supervision and Control System of metropolitan scope based on Public Communication Networks. $5^{\text {th }}$ IFAC Conference on Fieldbus Systems and their Applications. pp 317-323.

Zhou, L., P. Chan and R. Radhakrishna (2002). Effect of TCP/LLC protocol iteraction in GPRS networks. Computer Communications 25, pp 501-506.

3GPP (2002) Digital Cellular Telecomunication System (phase 2+); Overall description over the GPRS radio interface; Stage 2; (3GPP TS 43.064 version 5.0.0 Release 5). 